\title{
Multispectral 3D Whole-Body Imaging of Dressed and Undressed Bodies in Combination with Postmortem X-Ray Computed Tomography
}

\author{
Sören KOTTNER ${ }^{* 1}$, Martin M. SCHULZ ${ }^{2}$, Florian BERGER ${ }^{1}$, Michael J. THALI ${ }^{1}$, Dominic GASCHO ${ }^{1}$ \\ ${ }^{1}$ Zurich Institute of Forensic Medicine, University of Zurich, Zurich, Switzerland; \\ ${ }^{2}$ Institute of Forensic Medicine, Ludwig-Maximilians-University Munich, Munich, Germany
}

\section{Abstract}

https://doi.org/10.15221/21.24

\section{Introduction}

In the last few years, postmortem 3D body documentation, especially through the use of photogrammetry, has gained importance in the field of forensic medicine. For this purpose, conventional digital cameras are used to capture information within the visible part of the electromagnetic spectrum (i.e., visible light). Moreover, the use of multispectral photography allows the detection and documentation of traces and injuries, for instance, in the ultraviolet (UV) or near-infrared (NIR) range, which are otherwise invisible to the human eye. Although multispectral photography offers a wide range of applications for legal investigations, multispectral photogrammetry is not yet well known or widely used within the field of forensic medicine. Therefore, within this study and the framework of the VirtoScan project, a method for multispectral whole-body photogrammetry was developed and evaluated.

\section{Materials and Methods}

A multicamera setup based on four modified digital single-lens reflex cameras, different light sources, and additional lens filters was mounted on a mobile wooden frame. The setup was used in combination with a medical X-ray computed tomography (CT) scanner. Automatic table movement from the CT scanner was used to capture consecutive image sets of the body from head to toe. In addition to standard photogrammetry within the visible range, multispectral photogrammetry was performed under UV and NIR light sources at $365 \mathrm{~nm}, 400 \mathrm{~nm}, 860 \mathrm{~nm}$ and $960 \mathrm{~nm}$ on undressed human bodies and under blue light and NIR light sources at $450 \mathrm{~nm}$ and $860 \mathrm{~nm}$ on dressed mannequins. After the multispectral photogrammetry procedure was finished, a whole-body CT scan was conducted to capture the internal information of the human body.

\section{Results}

Multiview 3D reconstructions based on multispectral image data from four forensic cases and four different sets of dressed mannequins were carried out successfully. The overall quality and level of detail of the polygon models from the undressed bodies varied with regard to the spectral range of the image data. Dressed bodies captured under blue and visible light exhibited reduced quality and reduced level of detail on the polygon models within areas of dark-colored clothing. Whole-body photogrammetry for undressed bodies took approximately 5 min under UV illumination and approximately 3 min under visible light or NIR illumination. Whole-body photogrammetry for dressed bodies took approximately 12 min under blue-light illumination and approximately 4 min under visible light or NIR illumination.

\section{Discussion and Conclusion}

The multispectral camera setup allows the capture of whole body datasets in an extended spectral range within a few minutes. With the help of photogrammetry software, textured 3D models for different spectral ranges can be reconstructed. Multispectral 3D whole-body imaging in line with postmortem CT examinations allows the combination of multispectral information from external body documentation with radiological findings from internal body documentation. Multispectral 3D documentation extends the postmortem forensic documentation of the deceased, as it detects (and documents) latent evidence on the body and textiles and can assist in detecting subcutaneous injuries and bruises on the body.

Keywords: Photogrammetry, Infrared and Ultraviolet Photography, Multicamera Setup, 3D Body Scanning, Forensic Imaging, Legal Medicine, VirtoScan

\footnotetext{
*soeren.kottner@irm.uzh.ch
} 


\section{Introduction}

Within the last 24 years, the optical three-dimensional (3D) imaging of injuries and entire bodies has become increasingly used within the field of forensic medicine and has been proven to add beneficial value to forensic investigations [1]-[13]. In 1997, Brüschweiler et al. [1] demonstrated how the use of photogrammetry could be applied to forensic investigations to match an injury with the injury-causing object. Since then, a variety of optical 3D imaging systems have been used for the purpose of 3D injury and whole-body documentation. The use of optical 3D imaging has been shown to assist in 3D reconstructions of crime and accident scenes [7]-[9], [14]. Additionally, studies have presented workflows to combine optical $3 \mathrm{D}$ data with radiological datasets from medical $\mathrm{X}$-ray computed tomography (CT) [7], [8], [14]-[16]. Within recent years, single-camera and multicamera photogrammetry setups have been increasingly used for 3D whole-body imaging [17]-[20]. However, to date, the use of optical 3D imaging has mainly focused on the documentation of color information based on the visible part of the electromagnetic spectrum (i.e., visible light). Visible light corresponds to the spectral range of the electromagnetic spectrum that is visible to the human eye. Precise limits for the spectral range of visible light do not exist. The International Commission on Illumination (Commission Internationale de l'Eclairage - CIE, Vienna, Austria) has therefore defined the lower limit between $360 \mathrm{~nm}$ and $400 \mathrm{~nm}$ and the upper limit between $760 \mathrm{~nm}$ and $830 \mathrm{~nm}$ [21].

Although two-dimensional multispectral imaging, such as ultraviolet (UV) photography and near-infrared (NIR) photography, are established tools to detect, visualize and document evidence that is otherwise invisible to the naked eye, 3D multispectral imaging is not yet well known or commonly applied in this area. UV and NIR radiation are adjacent to the visible part of the electromagnetic spectrum. UV radiation is characterized by shorter wavelengths, ranging from $100 \mathrm{~nm}$ to $400 \mathrm{~nm}$ [21]. NIR radiation, in contrast, is characterized by longer wavelengths, ranging from $780 \mathrm{~nm}$ to $1400 \mathrm{~nm}$ [21]. An application that is most commonly associated with multispectral photography within the field of forensic science is the detection and visualization of latent bruises and injuries on the body [22]-[36]. Additionally, multispectral photography is commonly used for the detection and documentation of gunshot residue and bodily fluids on textiles and skin [37]-[44]. Apart from our recent publication [45], our literature research revealed that there is currently only one publication reporting the use of 3D multispectral photogrammetry of crime scenes [46]. Studies regarding the use of 3D multispectral imaging for forensic applications seem to be underrepresented within the scientific literature, whereas publications regarding 3D multispectral whole-body examinations of undressed bodies appear to be absent entirely.

To address the lack of information in this area, we aimed to build a simple and inexpensive multispectral 3D imaging setup for postmortem full-body examinations in combination with a medical CT scanner. The objective of this study was to investigate the feasibility of performing 3D multispectral imaging on dressed and undressed bodies using multicamera close-range photogrammetry. Furthermore, we aimed to test the applicability of 3D multispectral imaging of dressed bodies with selected stains (bodily fluids and spray paint) on white and black clothing.

\section{Materials and Methods}

\subsection{Imaging setup}

The 3D multispectral imaging setup was based on a multicamera rig that was used in conjunction with a medical CT scanner (Somatom Definition Flash, Siemens Healthineers, Erlangen, Germany). The construction and design of the setup are described in our previous reports [17], [19], [45]. The multicamera rig was composed of a wooden mobile frame carrying four modified digital single lens reflex (DSLR) cameras (EOS 200D, Canon Inc., Tokyo, Japan) in combination with $40 \mathrm{~mm}$ lenses (EF $40 \mathrm{~mm}$ $\mathrm{f} / 2.8 \mathrm{STM}$, Canon Inc.). Camera modification involved removal and replacement of the original camera filters in front of the sensor with a multispectral glass filter (transmission range: 190-4000 nm). The modification was carried out by a camera specialist (Optic Makario $\mathrm{GmbH}$, Mönchengladbach, Germany). Each camera was equipped with a remote shutter control (Yongnou RF-603C II, YongNuo Photographic Equipment Co., Ltd., Shenzhen, China) depending on the spectral range of the photogrammetry set and a specific set of lens filters and light sources (Tables 1-3). A photograph of the 3D multispectral imaging setup in combination with the CT scanner is shown in Fig. 1. A more detailed description of the setup can be found in our recent publication [45]. 


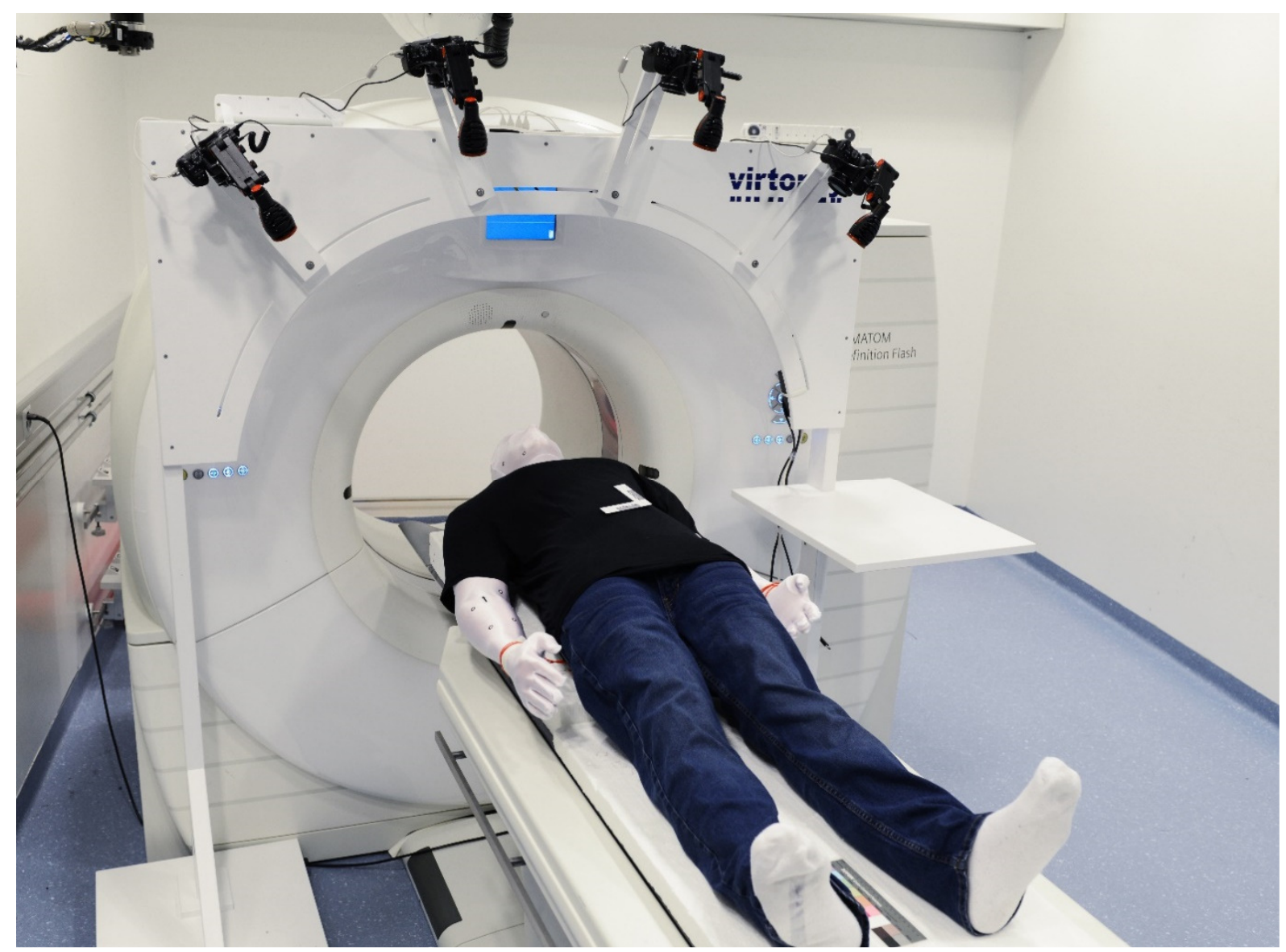

Fig. 1. Multispectral photogrammetry setup equipped with four modified DLSR cameras, NIR light sources, IR longpass filters and remote shutter controls. The setup is placed in front of a CT gantry and aligned centrally above a dressed mannequin.

Table 1. Lens filters used for multispectral photogrammetry. Detailed information about the 50\% transmission rate of IR neutralization filters is not available. According to the manufacturer, IR neutralization filters restore the original camera transmission range and color representation.

\begin{tabular}{llll}
\hline Manufacturer & Filter name & $\mathbf{5 0 \%}$ transmission [nm] & Approx. transmission range [nm] \\
\hline Optic Makario & yellow & 460 & $450-1100$ \\
Optic Makario & orange & 550 & $540-1100$ \\
Optic Makario & IR neutralization & N/A & Original camera transmission range \\
Optic Makario & IR longpass & 850 & $810-1100$ \\
\hline
\end{tabular}

Table 2. Tunable light sources used for multispectral photogrammetry.

\begin{tabular}{llll}
\hline Manufacturer & Product name & Wavelength [nm] & Class \\
\hline Dedolight & DLOBML-BI-UV, Fluoreszilla & $365-400$ & UV \\
Dedolight & DLOBML-BI-BB, LedZilla & $450-470$ & Blue light \\
Dedolight & DLOBML-BI-IR, iREDZILLA & $860-960$ & NIR \\
\hline
\end{tabular}




\subsection{Imaging workflow}

Multispectral whole-body photogrammetry was carried out semiautomatically. For image acquisition, the multicamera rig was first placed in front of the CT gantry and aligned centrally above the CT couch/body. Subsequently, image acquisition was carried out. The operating software of the CT scanner (Syngo CT 2012B, VA44A, Siemens Healthineers, Erlangen, Germany) was used to move the CT couch in consecutive steps underneath the multicamera rig. Between each step, the movement of the CT couch was stopped, and the cameras were triggered. This procedure was carried out for the entire length of the body. Multiple sets of whole-body photogrammetry involving different light sources and lens filters were needed to acquire a full 3D multispectral dataset of one body. For each photogrammetry set, the multicamera setup had to be adapted.

For the photogrammetry set within the visible spectrum, standard ceiling lighting was used as the light source, and an absorptive filter, called an IR neutralization filter, was attached to each camera lens. This filter restores the default transmission characteristics from the original camera configuration (Table 1). For the photogrammetry set based on NIR illumination, a tunable NIR light source with a range between 860-960 nm (DLOBML-BI-IR iREDZILLA, Dedolight Forensic Series, Dedo Weigert Film $\mathrm{GmbH}$ ) and a longpass filter with $50 \%$ transmission at $850 \mathrm{~nm}$ were attached to each camera. Undressed bodies were additionally captured under UV illumination in the range between 365-400 nm. UV illumination for undressed bodies was chosen in consultation with a forensic multispectral imaging specialist. For the photogrammetry sets based on UV illumination, a tunable UV light source (DLOBMLBI-UV Fluoreszilla, Dedolight Forensic Series, Dedo Weigert Film GmbH, Munich, Germany) and a yellow filter with $50 \%$ transmission at $460 \mathrm{~nm}$ were attached to each camera (Tables $1 \& 2$ ). Dressed bodies, in addition to the photogrammetry sets under visible light and NIR illumination at $860 \mathrm{~nm}$, were captured under blue-light illumination at $450 \mathrm{~nm}$. Previous tests showed that blue-light illumination at $450 \mathrm{~nm}$ and NIR illumination at $860 \mathrm{~nm}$ resulted in high-contrast visualizations of the selected stains. Depending on the stain (i.e., type of exemplary evidence), 3D multispectral datasets of dressed bodies were acquired under either visible light and blue-light illumination (urine and semen stains on white tshirts) or visible light and NIR illumination (blood stains and spray paint on black t-shirts). Details regarding the illumination setups are listed in Table 3 . For the photogrammetry sets based on UV, blue light and NIR illumination, any additional light source was turned off during the image-capturing procedure.

The camera settings for the aperture (f/16) and the signal gain (ISO 100) were maintained constant throughout the study. Exposure times, white balance and camera focus had to be adjusted manually for each photogrammetry set. Subsequent to the 3D multispectral whole-body photogrammetry of the undressed bodies, a whole-body CT scan was conducted to capture the internal information of the human body. In the case of dressed bodies, a mannequin was used instead of a human body. CT scans for dressed mannequins were omitted.

\subsection{D reconstruction and 3D model optimization}

After image acquisition, photogrammetric software for the multiview 3D reconstruction of the captured photogrammetric datasets was used (Agisoft Metashape Professional, Version 1.6.1 build 10009, Agisoft LLC, St. Petersburg). Each photogrammetry set was computed separately. The 3D reconstructions were computed based on default settings for high-resolution models. Textures were calculated based on a size of 12288 pixels. If the initial alignment of the photos failed or was incomplete, manual adjustments were made. Subsequent to the 3D reconstructions, all models were edited in 3D inspection software (GOM inspection suite, Version 2020, Hotfix 1, Rev. 131819, Build 2020-10-12, GOM GmbH, Braunschweig, Germany). The editing process involved cleaning the boundaries of the 3D mesh and erasing parts of the 3D model that held no useful information (e.g., the surface of the CT couch). This procedure helped reduce the overall size of the 3D datasets. Finally, with the help of Agisoft Metashape Professional, textures for the edited 3D models were calculated, and with the help of several point-to-point distances, all models were scaled to their true size. 
Table 3. Illumination setups used for the photogrammetric datasets of dressed (DB) and undressed bodies (UB).

\begin{tabular}{lcccccc}
\hline Datasets & \multicolumn{2}{c}{ UV } & Blue light & Visible light & \multicolumn{2}{c}{ NIR } \\
& $365 \mathbf{n m}$ & $\mathbf{4 0 0} \mathbf{n m}$ & $\mathbf{4 5 0} \mathbf{n m}$ & & $\mathbf{8 6 0} \mathbf{n m}$ & $\mathbf{9 6 0} \mathbf{n m}$ \\
\hline UB: all & $\mathrm{x}$ & $\mathrm{x}$ & - & $\mathrm{x}$ & $\mathrm{x}$ & $\mathrm{x}$ \\
DB: urine (white t-shirt) & - & - & $\mathrm{x}$ & $\mathrm{x}$ & - & - \\
DB: semen (white t-shirt) & - & - & $\mathrm{x}$ & $\mathrm{x}$ & - & - \\
DB: blood (black t-shirt) & - & - & - & $\mathrm{x}$ & $\mathrm{x}$ & - \\
DB: spray paint (black t-shirt) & - & - & - & $\mathrm{x}$ & $\mathrm{x}$ & - \\
\hline
\end{tabular}

\subsection{Forensic cases and selection of evidence for dressed mannequins}

Overall, four forensic cases (male: $n=1$; female: $n=3$ ) and four different sets of dressed mannequins were used to investigate the applicability of 3D multispectral full-body imaging. The forensic cases were selected randomly. The selection of exemplary evidence for the dressed mannequin (i.e., urine, semen, blood and black spray paint) was chosen based on applications for 2D multispectral imaging commonly found in the scientific literature. The urine and semen samples were applied to white cotton t-shirts (Table 3). The blood sample and black spray paint were applied to black cotton t-shirts (Table 3).

\section{Results}

Multiview 3D reconstructions based on multispectral image data from four forensic cases and four different sets of dressed mannequins were carried out successfully. For each case involving an undressed body, five photogrammetric datasets were recorded. For each case involving a dressed mannequin, two photogrammetric datasets were recorded. Altogether, 28 photogrammetric datasets were included in this study. On average, whole-body photogrammetry for undressed bodies took approximately $5 \mathrm{~min}$ under UV illumination and approximately $3 \mathrm{~min}$ under visible light or NIR illumination. Whole-body photogrammetry for dressed bodies took approximately 12 min under bluelight illumination and approximately 4 min under visible light or NIR illumination.

\subsection{Undressed bodies}

The overall quality and level of detail of the polygon models from the undressed bodies varied with regard to the spectral range of the image data. An example of the entire multispectral dataset for one of the forensic cases is given in Fig. 2. Images of the textured models (Fig. 2, a I-V) and their corresponding 3D polygonal mesh representation (Fig. 2, b I-V) are given. 3D polygonal meshes from the 3D reconstructions under visible light and UV illumination showed a comparable level of detail (Fig. 2, $\mathrm{b}$ I-III). 3D polygonal meshes from the 3D reconstructions under NIR illumination exhibited a higher level of noise (Fig. 2, b IV-V). 
a I

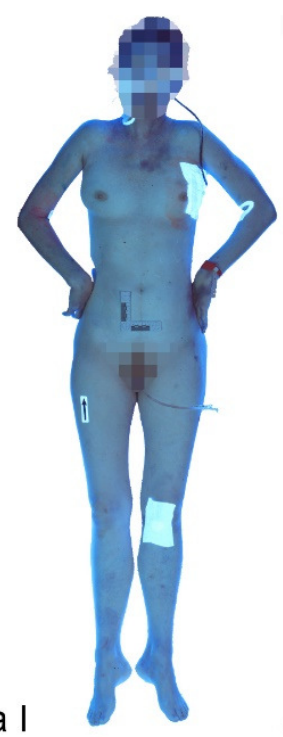

II

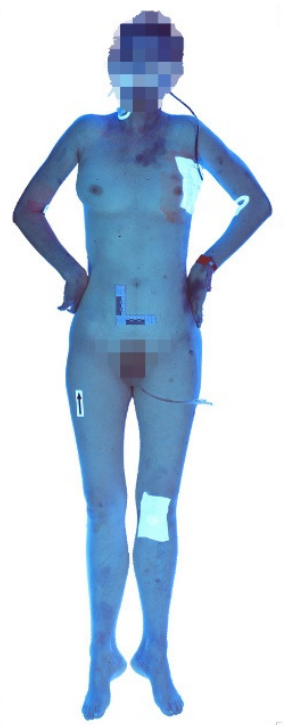

III
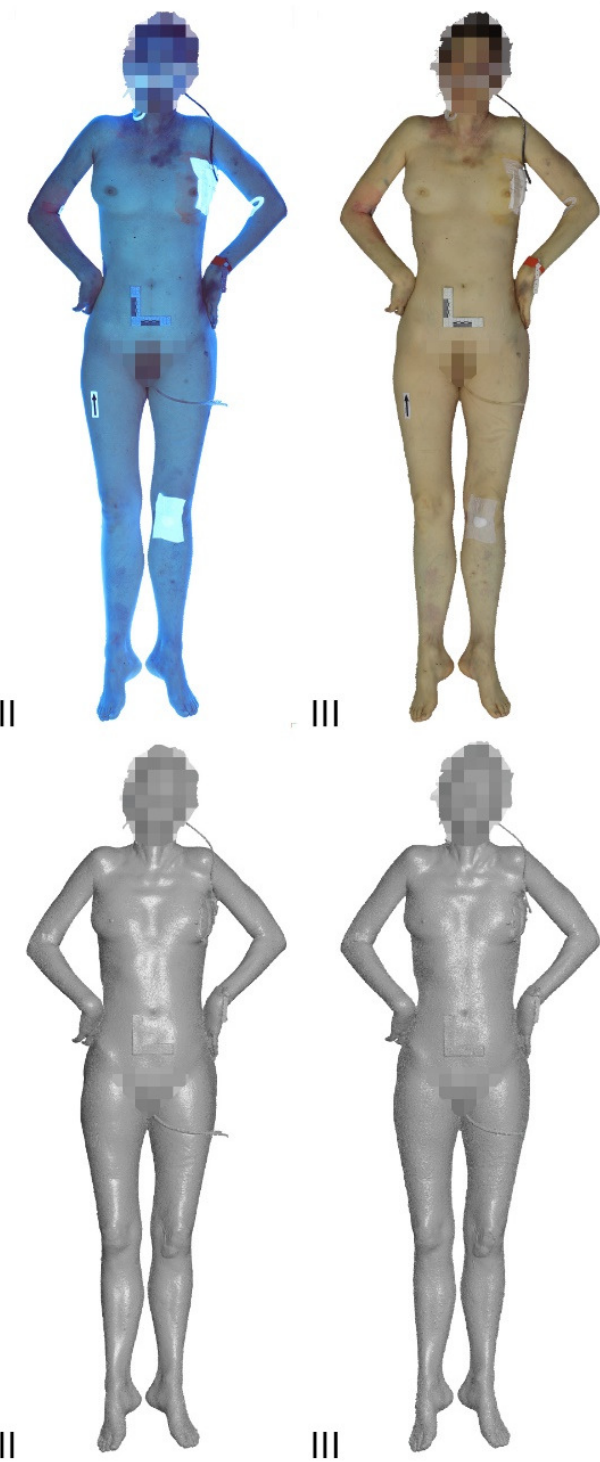
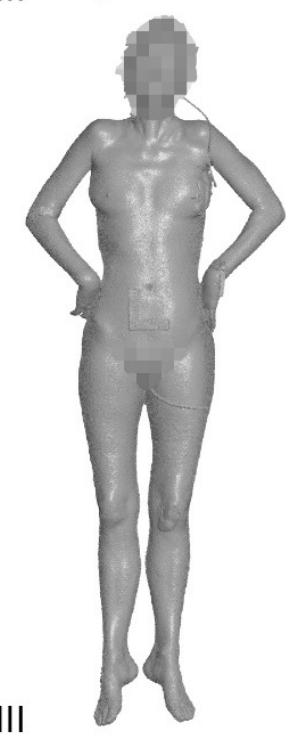

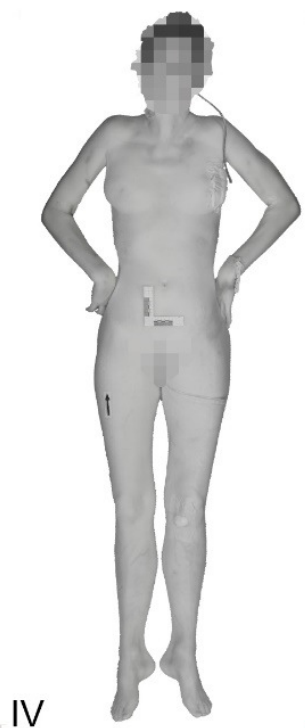

IV

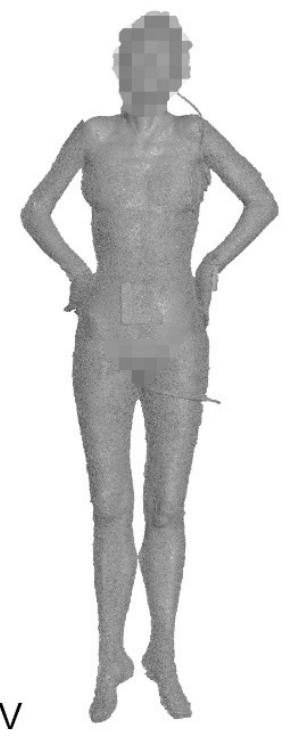

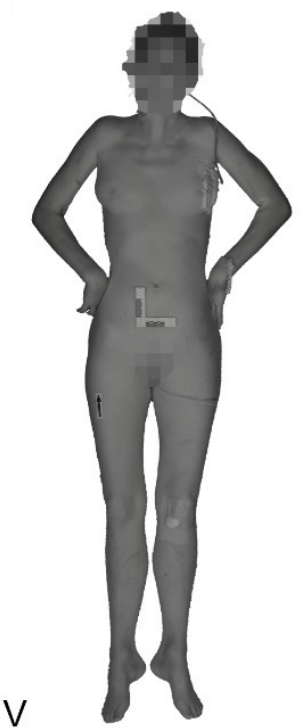

$\mathrm{V}$

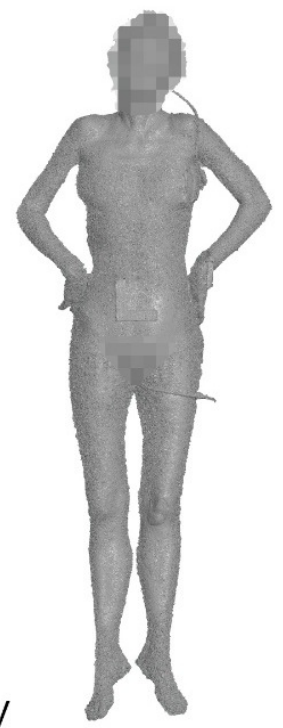

Fig. 2. Comparison of the textured $3 D$ models (a I-V) and the corresponding 3D polygonal mesh representations $(b \mathrm{I}-V$ ) from the entire multispectral dataset for one of the forensic cases. Images a I and $b$ I refer to the photogrammetric dataset under UV illumination at $365 \mathrm{~nm}$. Images a II and b II refer to the photogrammetric dataset under UV illumination at $400 \mathrm{~nm}$. The photogrammetric dataset under visible light is represented by images a III and $b$ III. The remaining image sets of a IV/b IV and a V/b V refer to the photogrammetric datasets under NIR illumination at $860 \mathrm{~nm}$ and $960 \mathrm{~nm}$, respectively.

Photogrammetric datasets under UV illumination compared to datasets under visible light showed an enhanced visibility of foreign substances on the skin. Substances that were found in the frame of this study appeared to be antiseptic cleansing agents, adhesives and bodily fluids. An example is given in Fig. 3. A trace of dried bodily fluid was visible on the photogrammetric dataset under UV illumination at $400 \mathrm{~nm}$ in combination with a yellow lens filter. In the corresponding photogrammetric datasets under visible light, the trace was initially not evident. A detailed view of the dataset, however, displayed a slight difference in surface texture that indicated the trace. Photogrammetric datasets under NIR illumination showed an enhanced visibility of vein patterns in the extremities. Datasets under visible light exhibited low contrast and weak visibility regarding vein patterns (Fig. 2, a III-V). Additionally, light-colored hematomas were detectable on the skin under visible light but were absent in the datasets under NIR illumination. Darker hematomas with a bluish appearance were evident under visible light and appeared with discernible contrast under NIR illumination. 

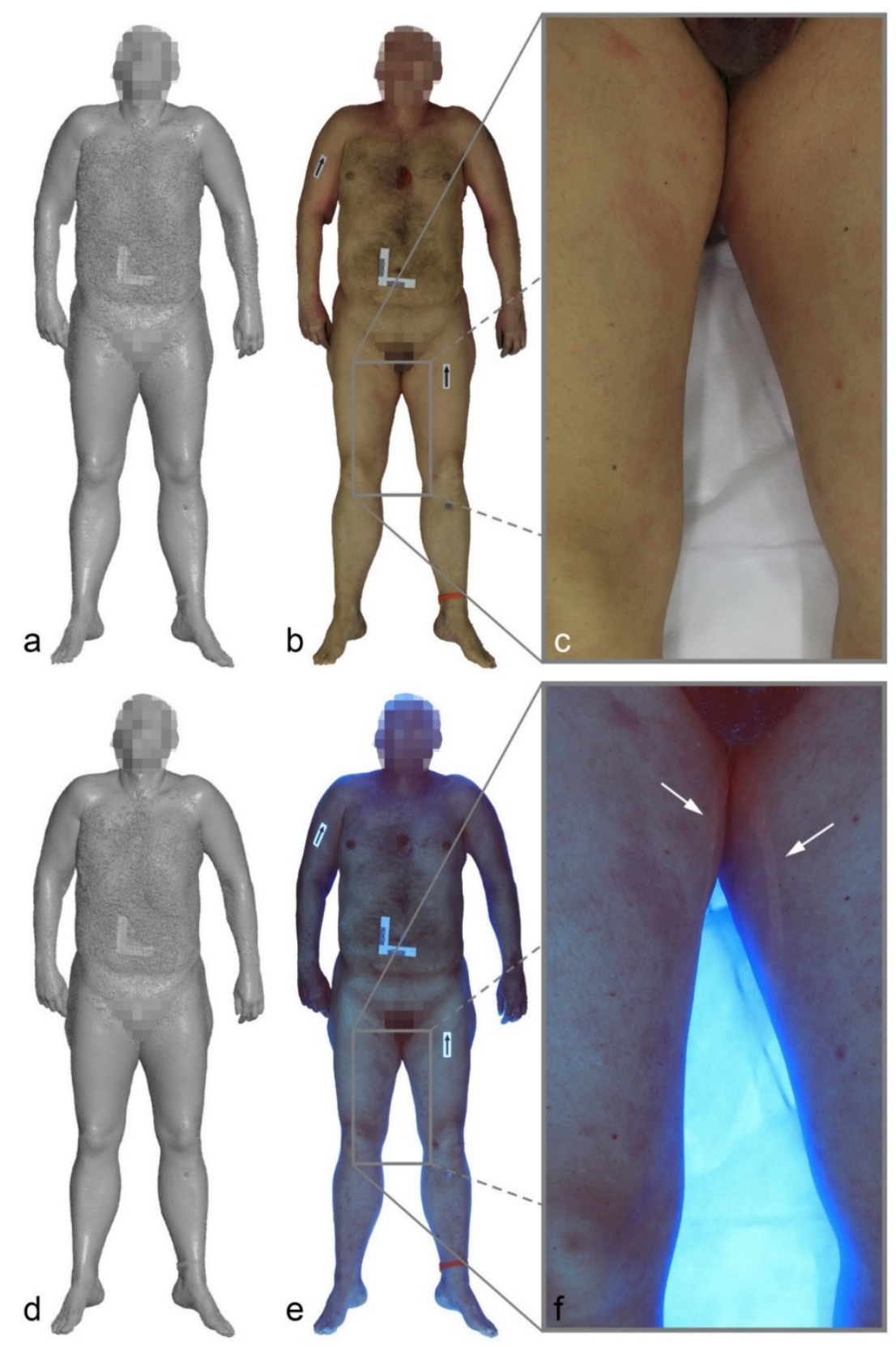

Fig. 3. 3D whole-body documentation under visible light and UV illumination. Images $a, b$ and c refer to the 3D data acquired under visible light. Images $d$, e, and f refer to the $3 D$ data acquired under UV illumination at 400 $\mathrm{nm}$. Images a and d illustrate the 3D mesh of the reconstructed 3D data. Images $b$ and e show the textured 3D model, whereas images $c$ and $f$ illustrate an enlarged view of the inner thigh. The arrows in image $f$ indicate a trace of fluorescent, dried bodily fluid.

\subsection{Dressed bodies}

The overall quality and level of detail of the polygon models from the dressed bodies varied with regard to the spectral range of the image data. Dressed bodies captured under visible light exhibited a higher level of noise and reduced quality within areas of black-colored clothing (i.e., black t-shirt). Dressed bodies captured under blue-light illumination showed a higher level of noise and a reduced level of detail within areas of dark-blue-colored clothing (i.e., blue jeans). The overall quality and level of detail from the polygon models under NIR illumination was good and largely consistent. An example of the $3 \mathrm{D}$ reconstructions from a dressed mannequin wearing a black cotton t-shirt is given in Fig. 4 . The polygonal mesh representations in combination with the textured 3D models and close-up views of the area covered by black spray paint are displayed for the photogrammetry sets under visible light (Fig. 4, a-c) and under NIR illumination (Fig. 4, d-f). 
The dried semen and dried urine stains on the white cotton t-shirts had weak visibility under visible light. With blue-light illumination, at $450 \mathrm{~nm}$ in combination with an orange lens filter, the stains stood out distinctively against the surrounding cotton fabric and appeared in a clearly visible fluorescent green color. Similarly, the dried blood stains and the dried black spray paint on the black cotton t-shirts were hardly visible under visible light (Fig. 4, b,c). With the use of NIR illumination at $860 \mathrm{~nm}$ in combination with an IR-longpass filter, however, the black t-shirt appeared as light gray, whereas the blood stain and the area covered by the black spray paint appeared as a darker gray with discernible contrast to the surrounding fabric (Fig. 4, e,f).
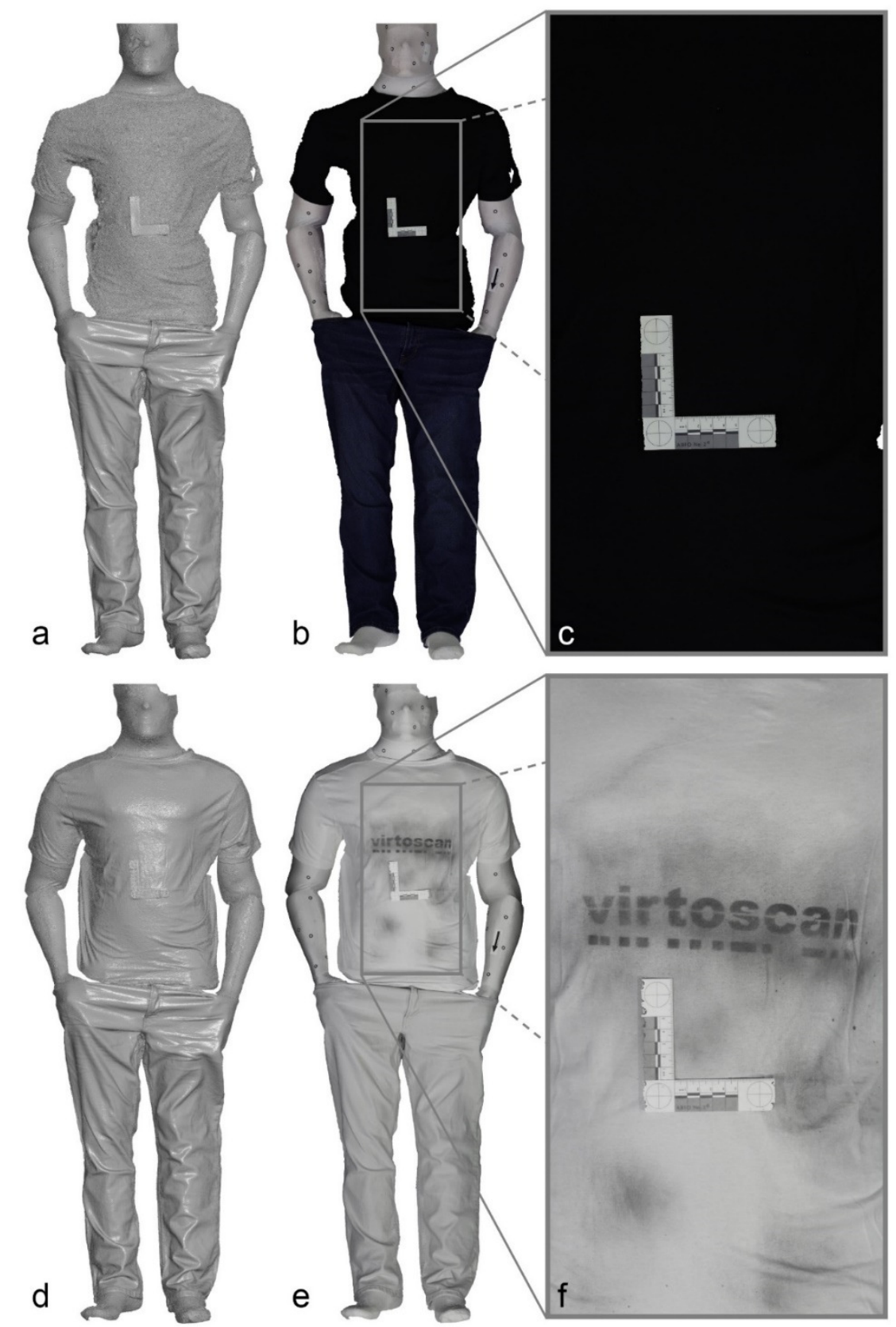

Fig. 4. 3D full-body imaging of a mannequin wearing a black $t$-shirt with black spray paint. Images $a, b$ and $c$ refer to the $3 D$ data acquired under visible light. Images $d$, e, and f refer to the $3 D$ data acquired under NIR illumination at $860 \mathrm{~nm}$. Images a and d illustrate the 3D mesh of the reconstructed 3D data. Images $b$ and e show the textured $3 D$ model, whereas images $c$ and fillustrate an enlarged view of the area covered by the spray paint. 


\section{Discussion}

In this study, a method for 3D multispectral whole-body imaging in conjunction with a medical CT scanner was developed and assessed. The imaging setup was based on a multicamera rig equipped with four modified DSLR cameras. 3D multispectral whole-body imaging was performed successfully on dressed and undressed bodies. The data quality of $3 \mathrm{D}$ reconstructions based on four forensic cases and four different sets of dressed mannequins was analyzed.

The results showed that the quality and the level of detail in the polygon models of the 3D reconstructions varied depending on the spectral range of the image data. 3D reconstructions for undressed bodies, for example, showed a comparable level of detail for the photogrammetry sets under visible light and UV illumination. In contrast, polygonal models based on the 3D reconstructions from the photogrammetry sets under NIR illumination were considerably lower in both quality and level of detail. The polygon models from the NIR datasets showed noisy 3D mesh representations featuring rough surfaces. 3D reconstructions for dressed bodies also showed that the quality and level of detail depended not only on the spectral range of the image data but also on textile color. Dressed bodies with dark-colored clothing (i.e., black t-shirt and blue jeans) seemed to perform well under NIR illumination. The level of detail for the reconstructed polygon models seemed good, and the quality appeared largely consistent across the entire 3D model. 3D reconstructions based on the photogrammetry sets under visible light and blue-light illumination, however, exhibited lower quality and a lower level of detail in areas of dark-colored clothing. Polygon models based on 3D reconstruction under visible light showed a reduced quality and a noisy 3D mesh representation on the parts of the 3D model that were covered by the black $\mathrm{t}$-shirt. Polygon models based on 3D reconstructions under blue light showed a reduced level of detail and a noisy mesh representation on the parts of the 3D model that were covered by the dark-blue-colored jeans.

These observations have in common that the lower-quality polygon models exhibited largely homogenous texture information in the original images. Images of undressed bodies under NIR illumination displayed a mainly uniform, light-gray texture. Images of black t-shirts under visible light appeared with a black homogenous texture, similar to the images of the dark-blue-colored jeans under blue-light illumination. Such homogenous textures and images are impractical for photogrammetric reconstructions that require nonuniform surfaces with sufficient textural information. Hence, the 3D reconstructions based on those images exhibited noisy and rough surfaces that contributed to a generally lower level of detail.

In addition to the analyses of the 3D reconstructions, features visualized or enhanced by multispectral 3D imaging in comparison to standard 3D imaging under visible light were analyzed. The results showed that 3D models of undressed bodies based on photogrammetry sets under UV illumination in combination with a yellow lens filter exhibited an enhanced visibility of foreign substances on the skin. In addition, injuries and bruises, which presumably were located deeper in the tissue, showed noticeable contrast in the textured 3D models based on photogrammetry sets under NIR illumination. In contrast, signs of subtle bruising on the skin could not be detected in the datasets under NIR illumination [45]. Furthermore, the results showed that 3D multispectral imaging could be used to enhance the visibility of certain substances on textiles. 3D models of dressed bodies based on photogrammetry sets under blue-light illumination in combination with an orange lens filter displayed an enhanced visibility of dried bodily fluids (i.e., semen and urine) on white t-shirts. In addition, dried blood stains and black spray paint on black t-shirts showed an enhanced contrast in the textured 3D models based on photogrammetry sets under NIR illumination. Overall, these findings seem consistent with the scientific literature.

This study demonstrated the possibility of using multispectral photogrammetry for postmortem wholebody documentation in conjunction with a medical CT scanner. This approach allows the 3D data to be aligned and merged with the corresponding CT data [7], [8], [14]-[16]. In the future, 3D multispectral imaging setups could be integrated into CT scanners to combine optical and radiological imaging to facilitate and accelerate the imaging workflow. However, the multicamera setup presented in this study is based on a movable rig and allows the manual capture of photogrammetric datasets. This setup can therefore be used in combination with any kind of examination couch or autopsy table to document postmortem and antemortem cases. Finally, 3D multispectral whole-body imaging setups could also be 
useful for application at the scene. In combination with mobile CT solutions based on customized trailers, mobile whole-body multispectral imaging facilities could contribute to legal investigations of violent crimes or during disaster victim identifications.

\section{Conclusion}

In this study, we showed that 3D multispectral imaging based on multicamera setups and close-range photogrammetry can be performed on dressed and undressed bodies for the detection, visualization, and documentation of forensic evidence. Furthermore, we introduced a simple and inexpensive multispectral imaging setup that can be used semiautomatically in conjunction with a medical CT scanner to perform 3D whole-body documentation of external and internal findings in human bodies.

\section{Acknowledgments}

The authors express their gratitude to Dr. Emma-Louise Kessler for her generous donation to the Zurich Institute of Forensic Medicine.

\section{Conflict of Interest}

Coauthor M.M. Schulz cooperates in an advisory capacity with Dedo Weigert Film GmbH, Munich, Germany. Products from Dedo Weigert Film GmbH used in this study were purchased from an official vendor. No products from Dedo Weigert Film GmbH were evaluated in this study.

\section{Ethical Approval}

The scan data were acquired as part of a forensic judicial investigation into a case. Anonymized results of these datasets are used in this publication. That data usage is conformant with Swiss laws and ethical standards. Ethical approval was waived by the Ethics Committee Zurich (KEK ZH-No. 15-0686).

\section{References}

[1] W. Brüschweiler, M. Braun, H. J. Fuchser, and R. Dirnhofer, "Photogrammetrische Auswertung von Haut- und Weichteilwunden sowie Knochenverletzungen zur Bestimmung des Tatwerkzeuges - grundlegende Aspekte," Rechtsmedizin, vol. 7, no. 3, pp. 76-83, https://doi.org/10.1007/BF03042360.

[2] M. J. Thali, M. Braun, W. Brüschweiler, and R. Dirnhofer, "Matching tire tracks on the head using forensic photogrammetry," Forensic Sci. Int., vol. 113, no. 1-3, pp. 281-287, Sep. 2000, https://doi.org/10.1016/S0379-0738(00)00234-6.

[3] W. Brüschweiler, M. Braun, R. Dirnhofer, and M. J. Thali, "Analysis of patterned injuries and injurycausing instruments with forensic 3D/CAD supported photogrammetry (FPHG): an instruction manual for the documentation process," Forensic Sci. Int., vol. 132, no. 2, pp. 130-138, Mar. 2003, https://doi.org/10.1016/S0379-0738(03)00006-9.

[4] M. J. Thali, M. Braun, W. Brueschweiler, and R. Dirnhofer, "'Morphological imprint': determination of the injury-causing weapon from the wound morphology using forensic 3D/CAD-supported photogrammetry," Forensic Sci. Int., vol. 132, no. 3, pp. 177-181, Apr. 2003, https://doi.org/10.1016/S0379-0738(03)00021-5.

[5] M. J. Thali et al., "Bite mark documentation and analysis: the forensic 3D/CAD supported photogrammetry approach," Forensic Sci. Int., vol. 135, no. 2, pp. 115-121, Aug. 2003, https://doi.org/10.1016/S0379-0738(03)00205-6.

[6] M. J. Thali, M. Braun, and R. Dirnhofer, "Optical 3D surface digitizing in forensic medicine: 3D documentation of skin and bone injuries," Forensic Sci. Int., vol. 137, no. 2-3, pp. 203-208, Nov. 2003, https://doi.org/10.1016/j.forsciint.2003.07.009. 
[7] U. Buck et al., "Application of 3D documentation and geometric reconstruction methods in traffic accident analysis: With high resolution surface scanning, radiological MSCT/MRI scanning and real data based animation," Forensic Sci. Int., vol. 170, no. 1, pp. 20-28, Jul. 2007, https://doi.org/10.1016/j.forsciint.2006.08.024.

[8] U. Buck, S. Naether, B. Räss, C. Jackowski, and M. J. Thali, "Accident or homicide - Virtual crime scene reconstruction using 3D methods," Forensic Sci. Int., vol. 225, no. 1-3, pp. 75-84, Feb. 2013, https://doi.org/10.1016/j.forsciint.2012.05.015.

[9] J. Tschui et al., "When the prey gets too big: an uncommon road accident involving a motorcyclist, a car and a bird," Int. J. Legal Med., vol. 130, no. 2, pp. 463-467, Apr. 2015, https://doi.org/10.1007/s00414-015-1188-0.

[10] C. Villa, "Forensic 3D documentation of skin injuries," Int. J. Legal Med., vol. 131, no. 3, pp. 751759, May 2017, https://doi.org/10.1007/s00414-016-1499-9.

[11] R. M. Carew and D. Errickson, "Imaging in forensic science: Five years on," J. Forensic Radiol. Imaging, vol. 16, pp. 24-33, Mar. 2019, https://doi.org/10.1016/j.jofri.2019.01.002.

[12] M. J. Flies, P. K. Larsen, N. Lynnerup, and C. Villa, "Forensic 3D documentation of skin injuries using photogrammetry: photographs vs video and manual vs automatic measurements," Int. J. Legal Med., vol. 133, no. 3, pp. 963-971, May 2019, https://doi.org/10.1007/s00414-018-1982-6.

[13] S. Kottner, P. M. Flach, D. Gascho, G. Ampanozi, M. Thali, and L. C. Ebert, "Communicating 3D data-interactive 3D PDF documents for expert reports and scientific publications in the field of forensic medicine," Int. J. Legal Med., vol. 134, no. 3, pp. 1175-1183, May 2020, https://doi.org/10.1007/s00414-019-02156-4.

[14] U. Buck, K. Buße, L. Campana, F. Gummel, C. Schyma, and C. Jackowski, "What happened before the run over? Morphometric 3D reconstruction," Forensic Sci. Int., vol. 306, p. 110059, Jan. 2020, https://doi.org/10.1016/j.forsciint.2019.110059.

[15] L. Campana, R. Breitbeck, R. Bauer-Kreuz, and U. Buck, "3D documentation and visualization of external injury findings by integration of simple photography in CT/MRI data sets (IprojeCT)," Int. J. Legal Med., vol. 130, no. 3, pp. 787-797, May 2016, https://doi.org/10.1007/s00414-015-12743.

[16] C. Villa, M. J. Flies, and C. Jacobsen, "Forensic 3D documentation of bodies: Simple and fast procedure for combining CT scanning with external photogrammetry data," J. Forensic Radiol. Imaging, vol. 12, pp. e2-e7, Mar. 2018, https://doi.org/10.1016/j.jofri.2017.11.003.

[17] S. Kottner, L. C. Ebert, G. Ampanozi, M. Braun, M. J. Thali, and D. Gascho, "A Mobile, Multi Camera Setup for 3D Full Body Imaging in Combination with Post-Mortem Computed Tomography Procedures," in Proceedings of the 7th International Conference on 3D Body Scanning Technologies, Lugano, Switzerland, 30 Nov.-1 Dec. 2016, pp. 53-60. https://doi.org/10.15221/16.053.

[18] A. Leipner, R. Baumeister, M. J. Thali, M. Braun, E. Dobler, and L. C. Ebert, "Multi-camera system for 3D forensic documentation," Forensic Sci. Int., vol. 261, pp. 123-128, Apr. 2016, https://doi.org/10.1016/j.forsciint.2016.02.003.

[19] S. Kottner, L. C. Ebert, G. Ampanozi, M. Braun, M. J. Thali, and D. Gascho, "VirtoScan - a mobile, low-cost photogrammetry setup for fast post-mortem 3D full-body documentations in $x$-ray computed tomography and autopsy suites," Forensic Sci. Med. Pathol., vol. 13, no. 1, pp. 34-43, Mar. 2017, https://doi.org/10.1007/s12024-016-9837-2.

[20] S. Kottner, S. Schaerli, M. Fürst, W. Ptacek, M. Thali, and D. Gascho, "VirtoScan-on-Rails - an automated 3D imaging system for fast post-mortem whole-body surface documentation at autopsy tables," Forensic Sci. Med. Pathol., vol. 15, no. 2, pp. 198-212, Jun. 2019, https://doi.org/10.1007/s12024-019-00095-5.

[21] International Commission on Illumination. CIE S 017/E:2020. In: ILV: International Lighting Vocabulary, 2nd Edition. Dec. 2020. https://doi.org/10.25039/S017.2020.

[22] J. A. Lovell, "Ultraviolet photography of injuries," Child Abuse Rev., vol. 2, no. 1, pp. 51-53, Mar. 1993, https://doi.org/10.1002/car.2380020110.

[23] E. Vogeley, M. C. Pierce, and G. Bertocci, "Experience With Wood Lamp Illumination and Digital Photography in the Documentation of Bruises on Human Skin," Arch. Pediatr. Adolesc. Med., vol. 156, no. 3, p. 265, Mar. 2002, https://doi.org/10.1001/archpedi.156.3.265. 
[24] F. D. Wright and G. S. Golden, "The use of full spectrum digital photography for evidence collection and preservation in cases involving forensic odontology," Forensic Sci. Int., vol. 201, no. 1-3, pp. 59-67, Sep. 2010, https://doi.org/10.1016/j.forsciint.2010.03.013.

[25] P. Rowan, M. Hill, G. A. Gresham, E. Goodall, and T. Moore, "The use of infrared aided photography in identification of sites of bruises after evidence of the bruise is absent to the naked eye," J. Forensic Leg. Med., vol. 17, no. 6, pp. 293-297, Aug. 2010, https://doi.org/10.1016/j.jlm.2010.04.007.

[26] G. S. Golden, "Standards and practices for bite mark photography," J. Forensic Odontostomatol., vol. 29, no. 2, pp. 29-37, Dec. 2011.

[27] D. S. Holbrook and M. C. Jackson, "Use of an Alternative Light Source to Assess Strangulation Victims," J. Forensic. Nurs., vol. 9, no. 3, pp. 140-145, Jul. 2013, https://doi.org/10.1097/JFN.0b013e31829beb1e.

[28] R. M. Limmen, M. Ceelen, U. J. L. Reijnders, S. Joris Stomp, K. C. de Keijzer, and K. Das, "Enhancing the Visibility of Injuries with Narrow-Banded Beams of Light within the Visible Light Spectrum," J. Forensic Sci., vol. 58, no. 2, pp. 518-522, Mar. 2013, https://doi.org/10.1111/15564029.12042.

[29] M. Lombardi, J. Canter, P. A. Patrick, and R. Altman, "Is Fluorescence Under an Alternate Light Source Sufficient to Accurately Diagnose Subclinical Bruising?," J. Forensic Sci., vol. 60, no. 2, pp. 444-449, Mar. 2015, https://doi.org/10.1111/1556-4029.12698.

[30] J. Glauche, B. Ondruschka, V. Wenzel, J. Dreßler, and C. Hädrich, "Examination of invisible injuries: UV radiation-induced fluorescence as a supplement to physical examination for blunt trauma injury," Rechtsmedizin, vol. 25, no. 6, pp. 543-547, Dec. 2015, https://doi.org/10.1007/s00194-015-0053-2.

[31] K. Olds, R. W. Byard, C. Winskog, and N. E. I. Langlois, "How useful are ultraviolet, infrared, and narrow band light sources for enhancing occult bruises in cases of assault?," Forensic Sci. Med. Pathol., vol. 12, no. 2, pp. 209-210, Jun. 2016, https://doi.org/10.1007/s12024-016-9756-2.

[32] K. Olds, R. W. Byard, C. Winskog, and N. E. I. Langlois, "Validation of ultraviolet, infrared, and narrow band light alternate light sources for detection of bruises in a pigskin model," Forensic Sci. Med. Pathol., vol. 12, no. 4, pp. 435-443, Dec. 2016, https://doi.org/10.1007/s12024-016-9813-x.

[33] K. Olds, R. W. Byard, C. Winskog, and N. E. I. Langlois, "Validation of alternate light sources for detection of bruises in non-embalmed and embalmed cadavers," Forensic Sci. Med. Pathol., vol. 13, no. 1, pp. 28-33, Mar. 2017, https://doi.org/10.1007/s12024-016-9822-9.

[34] T. Rost, N. Kalberer, and E. Scheurer, "A user-friendly technical set-up for infrared photography of forensic findings," Forensic Sci. Int., vol. 278, pp. 148-155, Sep. 2017, https://doi.org/10.1016/j.forsciint.2017.07.001.

[35] S. Mimasaka, T. Oshima, and M. Ohtani, "Visualization of old bruises in children: Use of violet light to record long-term bruises," Forensic Sci. Int., vol. 282, pp. 74-78, Jan. 2018, https://doi.org/10.1016/j.forsciint.2017.11.015.

[36] L. Trefan, C. Harris, S. Evans, D. Nuttall, S. Maguire, and A. M. Kemp, "A comparison of four different imaging modalities - Conventional, cross polarized, infra-red and ultra-violet in the assessment of childhood bruising," J. Forensic Leg. Med., vol. 59, pp. 30-35, Oct. 2018, https://doi.org/10.1016/j.jlm.2018.07.015.

[37] V. Barrera, B. Fliss, S. Panzer, and S. A. Bolliger, "Gunshot residue on dark materials: a comparison between infrared photography and the use of an alternative light source," Int. J. Legal Med., vol. 133, no. 4, pp. 1115-1120, Jul. 2019, https://doi.org/10.1007/s00414-018-1965-7.

[38] K. A. Santucci, D. G. Nelson, K. K. McQuillen, S. J. Duffy, and J. G. Linakis, "Wood's Lamp Utility in the Identification of Semen," PEDIATRICS, vol. 104, no. 6, pp. 1342-1344, Dec. 1999, https://doi.org/10.1542/peds.104.6.1342.

[39] D. G. Nelson, "An Alternate Light Source to Detect Semen," Acad. Emerg. Med., vol. 9, no. 10, pp. 1045-1048, Oct. 2002, https://doi.org/10.1197/aemj.9.10.1045.

[40] C. Carter-Snell and K. Soltys, "Forensic Ultraviolet Lights in Clinical Practice: Evidence for the Evidence," vol. 3, no. 2, p. 7, 2005.

[41] A. C.-Y. Lin, H.-M. Hsieh, L.-C. Tsai, A. Linacre, and J. C.-I. Lee, "Forensic Applications of Infrared Imaging for the Detection and Recording of Latent Evidence," J. Forensic Sci., vol. 52, no. 5, pp. 1148-1150, Sep. 2007, https://doi.org/10.1111/j.1556-4029.2007.00502.x. 
[42] T. Gabby, "Sexual Abuse of Children: The Detection of Semen on Skin," Am. J. Dis. Child., vol. 146, no. 6, p. 700, Jun. 1992, https://doi.org/10.1001/archpedi.1992.02160180058017.

[43] J. Wawryk and M. Odell, "Fluorescent identification of biological and other stains on skin by the use of alternative light sources," J. Clin. Forensic Med., vol. 12, no. 6, pp. 296-301, Dec. 2005, https://doi.org/10.1016/j.jcfm.2005.03.005.

[44] C. A. Lincoln, P. M. McBride, G. R. Turbett, C. D. Garbin, and E. J. MacDonald, "The use of an alternative light source to detect semen in clinical forensic medical practice," J. Clin. Forensic Med., vol. 13, no. 4, pp. 215-218, May 2006, https://doi.org/10.1016/j.jcfm.2006.02.016.

[45] S. Kottner, M. M. Schulz, F. Berger, M. Thali, and D. Gascho, "Beyond the visible spectrum applying 3D multispectral full-body imaging to the VirtoScan system," Forensic Sci. Med. Pathol., Sep. 2021, https://doi.org/10.1007/s12024-021-00420-x.

[46] G. J. Edelman and M. C. Aalders, "Photogrammetry using visible, infrared, hyperspectral and thermal imaging of crime scenes," Forensic Sci. Int., vol. 292, pp. 181-189, Nov. 2018, https://doi.org/10.1016/j.forsciint.2018.09.025. 